On the relation between the Einstein field equations and the Jacobi-Ricci-Bianchi system

This article has been downloaded from IOPscience. Please scroll down to see the full text article.

2013 Class. Quantum Grav. 30145010

(http://iopscience.iop.org/0264-9381/30/14/145010)

View the table of contents for this issue, or go to the journal homepage for more

Download details:

IP Address: 87.64.179.242

The article was downloaded on 15/06/2013 at 10:30

Please note that terms and conditions apply. 


\title{
On the relation between the Einstein field equations and the Jacobi-Ricci-Bianchi system
}

\author{
N Van den Bergh \\ Department of Mathematical Analysis EA16, Ghent University, Galglaan 2, B-9000 Ghent, \\ Belgium \\ E-mail: norbert.vandenbergh@ugent.be
}

Received 18 March 2013, in final form 23 May 2013

Published 14 June 2013

Online at stacks.iop.org/CQG/30/145010

\begin{abstract}
The $1+3$ covariant equations, embedded in an extended tetrad formalism and describing a spacetime with an arbitrary energy-momentum distribution, are reconsidered. It is shown that, provided the $1+3$ splitting is performed with respect to a generic time-like congruence with a tangent vector $\boldsymbol{u}$, the Einstein field equations can be regarded as the integrability conditions for the Jacobi and Bianchi equations together with the Ricci equations for $\boldsymbol{u}$. The same conclusion holds for a generic null congruence in the Newman-Penrose framework.
\end{abstract}

PACS number: 04.20.Cv

\section{Introduction}

Although the Einstein field equations ${ }^{1}$

$$
R_{i j}-\frac{1}{2} R g_{i j}=T_{i j}
$$

have a deceptively simple appearance, when written out in terms of coordinates and the components of the metric, they form an unwieldy set of second-order nonlinear partial differential equations. This is one of the reasons why, during the past half century and in several areas of gravity research (explicit construction and classification of exact solutions [18, 19], cosmological perturbations and gravitational waves [16, 4, 12, 31], numerical relativity $[17,15,36,33,30]$, fundamental aspects $[5,3,29,1,2])$, focus has been shifting towards tetrad formulations of the theory. In this paper, I consider the so-called covariant $1+3$ formalism and the related orthonormal tetrad formalism, which continue to play an important role in the context of cosmology, as well as the Newman-Penrose formalism. The structure of the governing equations of relativistic cosmology, including their consistency, in both formalisms has by now been discussed in a large number of papers [9, 23, 20, 21, 34, 37, 32, 38] (for a recent review see the book by Ellis et al [13]), while the integrability of the equations

1 As our discussion applies to a completely general energy-momentum tensor, a possibly non-zero cosmological constant has been observed in the latter. 
in general tetrad formalisms has been discussed in detail by Edgar [7, 8] and MacCallum [24], following earlier work by Papapetrou [25-27]. There still remain some question marks regarding the redundancies present in these formalisms: while it is obvious that there are a number of algebraic interdependences, the issue of differential relations (more particularly the possibility of obtaining certain equations as integrability conditions of others) is less clear. This paper aims to have a closer look at these differential interdependences among the sets of Jacobi, Ricci and Bianchi equations on the one hand and the field equations on the other. As the commutator relations form a natural ingredient of such an investigation, the Jacobi equations will play a prominent role. I first repeat some basic facts about tetrad formalisms in section 2, closely following paper [24] and pointing out some subtleties with regard to the Jacobi equations. In section 3, I show that the field equations turn out to be the integrability conditions of the Jacobi-Ricci-Bianchi (JRB) set, provided the time-like congruence, with respect to which the $1+3$ splitting is carried out, has a non-vanishing acceleration which is not an eigenvector of $\sigma_{a b}+\omega_{a b}$. In section 4, I show that the same conclusion holds for a null congruence $\boldsymbol{k}$, provided that in a Newman-Penrose tetrad $(\boldsymbol{m}, \overline{\boldsymbol{m}}, \boldsymbol{\ell}, \boldsymbol{k})$ the null congruence $\boldsymbol{k}$ satisfies $-\kappa \equiv k_{a ; b} k^{b} m^{a} \neq 0$.

\section{Tetrad formalisms}

As usual spacetime is represented by a pseudo-Riemannian manifold and all calculations apply to an open set of spacetime. Notations and conventions are as in [19]. A $1+3$ splitting is accomplished locally by choosing a congruence of time-like worldlines with a unit tangent vector field $\boldsymbol{u}$. This congruence may be interpreted as the 4-velocity field of a family of observers, but no further restrictions apply: the congruence not necessarily consists of trajectories orthogonal to some family of space-like hypersurfaces, neither needs the unit tangent vector field to be parallelly transported or be invariantly defined (although in most applications one of these situations might occur). A set of (smooth) basis vector fields is then constructed by erecting at each point a spatial triad $\partial_{\alpha}$ orthogonal to $\partial_{0}=\mathbf{u}$ (note that $\partial_{a}$ are not partial derivatives, but frame derivatives $\left.{ }^{2}: \partial_{a}=e_{a}{ }^{i} \partial / \partial x^{i}\right)$. Denoting the dual basis of 1 -forms as $\omega^{a}=\omega^{a}{ }_{i} \mathrm{~d} x^{i}$ and the Levi-Civita connection as $\nabla$, the connection coefficients and connection 1-forms are given by $\nabla_{\partial_{a}} \partial_{b}=\Gamma_{b a}^{c} \partial_{c}$ and $\Gamma_{b}^{a}=\Gamma_{b c}^{a} \omega^{c}$, respectively. The Cartan structure equations read then

$$
\mathrm{d} \omega^{a}=-\Gamma_{b}^{a} \wedge \omega^{b}
$$

and

$$
\mathrm{d} \boldsymbol{\Gamma}_{b}^{a}+\Gamma_{c}^{a} \wedge \Gamma_{b}^{c}=\boldsymbol{R}_{b}^{a},
$$

with $\boldsymbol{R}_{b}^{a}$ being the curvature 2-forms. The integrability conditions for (2) and (3) are given by the first and second Bianchi identities, $\mathrm{d}^{2} \omega^{a}=0$ and $\mathrm{d}^{2} \boldsymbol{\Gamma}^{a}{ }_{b}=0$, or

$$
\begin{aligned}
& \boldsymbol{R}^{a}{ }_{c} \wedge \omega^{c}=0, \\
& \mathrm{~d} \boldsymbol{R}^{a}{ }_{b}-\boldsymbol{R}^{a}{ }_{c} \wedge \Gamma^{c}{ }_{b}+\Gamma^{a}{ }_{c} \wedge \boldsymbol{R}^{c}{ }_{b}=0 .
\end{aligned}
$$

Henceforth, it will be assumed, as is usually done in tetrad formulations of general relativity, that the basis is rigid, in the sense that the metric components $g_{a b}$ are constants,

$$
\mathrm{d} g_{a b}=\mathrm{d}\left(\partial_{a} . \partial_{b}\right)=0
$$

2 Indices $a, b, c, \ldots$ and $i, j, k, \ldots$ are respectively frame indices and coordinate indices taking the values $0,1,2,3$; greek indices $\alpha, \beta, \gamma, \ldots$ are frame indices taking the values $1,2,3$. 
with, for an orthonormal tetrad, $g_{a b}=\operatorname{diag}(-1,+1,+1,+1)$. Raising and lowering tetrad indices with $g_{a b}$ and its inverse and defining $\Gamma_{a b}=g_{a c} \Gamma^{c}{ }_{b}(6)$ implies $\boldsymbol{\Gamma}_{a b}=\boldsymbol{\Gamma}_{[a b]}$ or

$$
\Gamma_{(a b) c}=0,
$$

such that (2) completely defines the connection.

The commutation coefficients $\gamma_{a b}^{c}$ are defined by

$$
\left[\partial_{a}, \partial_{b}\right]=\gamma_{a b}^{c} \partial_{c},
$$

or

$$
\left.\gamma_{a b}^{c}=2 \omega^{c}{ }_{j} e_{[b}{ }^{j}, a\right],
$$

with a comma denoting a partial derivative or a frame derivative, depending on the index used. The first Cartan equations (2) express that the connection is torsion-free and hence relate the connection and commutation coefficients by

$$
\gamma_{a b}^{c}=2 \Gamma_{[b a]}^{c},
$$

which, for a rigid basis, by (7) is equivalent to

$$
\Gamma_{c a b}=\frac{1}{2}\left(\gamma_{b c a}+\gamma_{a c b}-\gamma_{c a b}\right)
$$

Introducing the components $R_{b c d}^{a}$ of the curvature 2-forms by

$$
\boldsymbol{R}_{b}^{a}=\frac{1}{2} R^{a}{ }_{b c d} \boldsymbol{\omega}^{c} \wedge \boldsymbol{\omega}^{d}
$$

the second Cartan equation becomes

$$
R_{b c d}^{a}=\Gamma_{b d, c}^{a}-\Gamma_{b c, d}^{a}+\Gamma_{b d}^{e} \Gamma_{e c}^{a}-\Gamma_{b c}^{e} \Gamma_{e d}^{a}-\gamma_{c d}^{e} \Gamma_{b e}^{a} .
$$

The first Bianchi identity (4), which can also be written as

$$
R_{a[b c d]}=0,
$$

is then seen to be equivalent to the Jacobi identity for a triple of basis vectors $\partial_{a}$,

$$
\left[\partial_{[a},\left[\partial_{b}, \partial_{c}\right]\right]=0,
$$

or

$$
\partial_{[a} \gamma_{b c]}^{d}-\gamma_{e[a}^{d} \gamma_{b c]}^{e}=0
$$

while the second Bianchi identity (5) can be written as (a semi-colon denoting the covariant derivative with respect to $\nabla$ )

$$
R_{b[c d ; e]}^{a}=0 .
$$

In a (pseudo-)Riemannian space, the Riemann tensor also must satisfy

$$
R_{a b c d}=-R_{b a c d}=-R_{a b d c}=R_{c d a b} .
$$

There is some confusion about whether or not these symmetry conditions have to be imposed as extra conditions [24]: if the connection is defined in terms of the commutator coefficients by (11) and the Riemann tensor is defined by (13), then $R_{a b c d}=g_{a e} R_{b c d}^{e}$ is automatically anti-symmetric in the first and second pair of indices. Provided that the Jacobi equations (16) hold, this implies also $R_{a b c d}=R_{c d a b}$. This is what happens in a so-called minimal tetrad formulation $[7,13]^{3}$, where one regards the tetrad components $e_{a}{ }^{i}$, the $\gamma_{a b}^{c}$ and the matter fields as functions to be solved for, with the governing equations being the component forms (9) and (16) of respectively the first Cartan and Jacobi equations and the field equations (1) together with the matter field equations. Note the crucial role of the Jacobi equations, which,

3 The versions in the cited works differ slightly. 
via the symmetry conditions (18), also imply that the Ricci tensor, defined by contraction of (13),

$$
R_{b d}=R_{b c d}^{c}=i_{\partial_{b}} i_{\partial_{m}} \boldsymbol{R}_{d}^{m},
$$

is symmetric. In addition, the Einstein tensor is then divergence-free, such that the matter field equations must be compatible with $T_{; b}^{a b}=0$. In this approach, the remaining trace-free part of the curvature is given by the Weyl tensor, which is defined by

$$
C_{a b c d}=R_{a b c d}-\left(g_{a[c} R_{d] b}+g_{b[d} R_{c] a}\right)+\frac{1}{3} R g_{a[c} g_{d] b} .
$$

As noted in [7], one can remove the tetrad components $e_{a}{ }^{i}$ from the system and view the operators $\partial_{a}$ as the third set of variables alongside the $\gamma_{a b}^{c}$ and the matter fields. The governing equations are then the commutator relations (8), formally describing the behaviour of the $\partial_{a}$ 'variables', the Jacobi equations (16) and the field equations (1) together with the matter field equations.

Because of the special role played by the Weyl tensor in the construction of exact solutions and the classification of spacetime geometries, it has become customary to include the components of the Weyl tensor as extra variables in the previous system. In the case of an orthonormal tetrad formalism, one decomposes then the Weyl tensor with respect to a time-like congruence defined by $\boldsymbol{u}=\partial_{0}$ into its electric and magnetic components ${ }^{4}$,

$$
C_{a b}^{c d}=4\left(u_{[a} u^{[c}+h_{[a}^{[c}\right) E_{b]}^{d]}+2 \varepsilon_{a b e} u^{[c} H^{d] e}+2 \varepsilon^{c d e} u_{[a} H_{b] e},
$$

or

$$
\begin{aligned}
& E_{a b}=C_{a b c d} u^{c} u^{d}, \\
& H_{a b}=\frac{1}{2} \eta_{a c}{ }^{e f} C_{e f b d} u^{c} u^{d} .
\end{aligned}
$$

Provided the Jacobi equations hold and the Riemann tensor is defined by (13), both $E_{a b}$ and $H_{a b}$ are then symmetric and trace-free.

Alternatively, one can set up an extended tetrad formalism [13], in which the fundamental variables are the $\partial_{a}$ operators, the $\gamma_{a b}^{c}$ coefficients and the symmetric tensors $E_{a b}, H_{a b}, T_{a b}$ ( $E_{a b}$ and $H_{a b}$ being also trace-free) and the matter fields. It is furthermore customary to split $T_{a b}$ as

$$
T_{a b}=\rho u_{a} u_{b}+p h_{a b}+2 q_{(a} u_{b)}+\pi_{a b},
$$

where the energy current density vector $q_{a}$ and the anisotropic pressure tensor $\pi_{a b}=\pi_{(a b)}$ are orthogonal to $u_{a}$ and $\pi_{a}^{a}=0$. In this extended formalism, the Riemann tensor is defined by (20), using (1) and (21) to express it in terms of the variables $E_{a b}, H_{a b}, \pi_{a b}, q_{a}, \rho$ and $p^{5}$. The symmetry and trace properties of these variables guarantee conditions (14), (18), and the governing equations become the second Bianchi equations (17) (written in terms of $E_{a b}, H_{a b}$, $\pi_{a b}, q_{a}, \rho, p$ and their covariant derivatives) and (13), implying (16). Note that (13) is now a set of partial differential equations for the $\gamma^{a}{ }_{b c}$ and not a definition for the Weyl tensor and that its contraction automatically yields the Einstein field equations (1). An equivalent approach is to implement (13) by requiring that the Ricci equation holds, when applied to all four of the basis vector fields:

$$
w_{; c ; d}^{a}-w_{; d ; c}^{a}=R_{b d c}^{a} w^{b},
$$

again with appropriate substitutions for the Riemann tensor in terms of $E_{a b}, H_{a b}$ and $T_{a b}$.

4 The Levi-Civita tensor $\boldsymbol{\eta}$ is normalized such that (tetrad indices!) $\eta_{0123}=-1$; we also define $\varepsilon_{a b c}=\eta_{a b c d} u^{d}$, while $h_{a b}$ is the projector in the instantaneous 3-spaces orthogonal to $\boldsymbol{u}$, defined by $h_{a b}=g_{a b}+u_{a} u_{b}$.

5 For example: $R_{1214}=H_{31}-\frac{1}{2} q_{2}, R_{1212}=\frac{1}{3} \rho-\frac{1}{2} \pi_{33}-E_{33}, \ldots$ 
A third approach to implement (13) (which is the one usually followed in cosmological applications, see for example [33, 32] or section 6.6 of [13]) is to impose only the Ricci equation applied to $\boldsymbol{u}=\partial_{0}$,

$$
u_{; c ; d}^{a}-u_{; d ; c}^{a}=R_{0 d c}^{a}
$$

and to add the remaining field equations (1), together with the Bianchi equations (17) and the Jacobi equations (16) (which now not automatically hold, as only part of the Ricci equations is used). The variables of the resulting system, which obviously contains a number of redundancies, can be written explicitly ${ }^{6}$ as $\dot{u}_{\alpha}, \omega_{\alpha}, \theta, \sigma_{\alpha \beta}, a_{\alpha}, n_{\alpha \beta}, \Omega_{\alpha}, \rho, p, q_{\alpha}, \pi_{\alpha \beta}, E_{\alpha \beta}$ and $H_{\alpha \beta}$, with the kinematical quantities $\dot{u}_{\alpha}, \omega_{\alpha}=\frac{1}{2} \varepsilon_{\alpha \beta \gamma} \omega^{\beta \gamma}, \theta$ and $\sigma_{\alpha \beta}$ being defined in the usual way by splitting $u_{a ; b}$ as

$$
u_{a ; b}=-\dot{u}_{a} u_{b}+\sigma_{a b}+\frac{1}{3} \theta h_{a b}+\omega_{a b}
$$

with

$$
\sigma_{a b}=\sigma_{(a b)}, \quad \sigma_{a}^{a}=0, \quad \omega_{a b}=\omega_{[a b]}, \quad \sigma_{a b} u^{b}=\omega_{a b} u^{b}=\dot{u}_{a} u^{a}=0 .
$$

$\Omega^{a}=\frac{1}{2} \eta^{a b c d} u_{b} \partial_{c} \cdot \dot{\partial}_{d}$ is the local angular velocity, in the rest frame of an observer with 4velocity $u$, of the triad $\partial_{\alpha}$ with respect to a set of Fermi-propagated axes (using MacCallum's convention [23]) and $n_{\alpha \beta}, a_{\alpha}$ are the Kundt-Schücking-Behr variables [10] parametrizing the purely spatial commutation coefficients $\gamma_{\beta \gamma}^{\alpha}$. The commutation coefficients can be read off from

$$
\begin{aligned}
& {\left[\partial_{0}, \partial_{\alpha}\right]=\dot{u}^{\alpha} \partial_{0}-\left(\frac{1}{3} \theta \delta_{\alpha}^{\beta}+\sigma_{\alpha}^{\beta}+\varepsilon_{\alpha \gamma}^{\beta}\left(\omega^{\gamma}+\Omega^{\gamma}\right)\right) \partial_{\beta},} \\
& {\left[\partial_{\alpha}, \partial_{\beta}\right]=-2 \varepsilon_{\alpha \beta \gamma} \omega^{\gamma} \partial_{0}+\left(2 a_{[\alpha} \delta_{\beta]}^{\gamma}+\varepsilon_{\alpha \beta \delta} n^{\delta \gamma}\right) \partial_{\gamma}}
\end{aligned}
$$

and the connection 1-forms read accordingly ${ }^{7}$

$$
\begin{aligned}
& \boldsymbol{\Gamma}_{10}=\dot{u}_{1} \boldsymbol{\omega}^{0}+\theta_{1} \boldsymbol{\omega}^{1}+\left(\sigma_{12}+\omega_{3}\right) \boldsymbol{\omega}^{2}+\left(\sigma_{13}-\omega_{2}\right) \boldsymbol{\omega}^{3} \\
& \boldsymbol{\Gamma}_{12}=-\Omega_{3} \boldsymbol{\omega}^{0}+\left(n_{13}-a_{2}\right) \boldsymbol{\omega}^{1}+\left(n_{23}+a_{1}\right) \boldsymbol{\omega}^{2}+\frac{1}{2}\left(n_{33}-n_{11}-n_{22}\right) \boldsymbol{\omega}^{3} .
\end{aligned}
$$

In cosmological applications, it is furthermore customary to define a fully projected covariant derivative $\tilde{\nabla}$, having the property that for an arbitrary tensor $S$

$$
\tilde{\nabla}_{a} S^{c \ldots d}{ }_{e \ldots f}=h_{a}{ }^{b} h_{p}^{c}{ }_{p} \ldots h^{d}{ }_{q} h_{e}{ }^{r} \ldots h_{f}{ }^{s} S^{p \ldots q}{ }_{r \ldots s ; b} .
$$

This allows one-at least formally - to remove the $n_{\alpha \beta}$ and $a_{\alpha}$ variables from a subset of equations (1), (16), (17) and (26), resulting in a set consisting of 4 Jacobi equations, 4 ( $0 a$ ) Einstein field equations, 10 Ricci equations (26) and the 20 Bianchi equations (17) and which is usually referred to as the ' $1+3$ covariant equations'. This system is quite elegant, bears a strong analogy with the Maxwell equations [22] (for a critique of this analogy see however [6]) and forms the basis of a number of significant contributions to cosmology. It is however an incomplete system and the fact that extra spatial information, representing the 12 remaining Jacobi equations and 6 Einstein field equations, must be added in one way or another has been reported on several occasions [11,24,37,33,32]. This incompleteness problem, in combination with the redundancies present in the fully extended system when the missing Einstein field equations are added by hand, was one of the main motivations for the present investigation. It turns out that (see section 3) when the time-like congruence corresponding to $\boldsymbol{u}$ is generic, adding the six spatial field equations is not necessary at all. In fact, we will do more and show

\footnotetext{
6 As explained above also the $\partial_{a}$ operators themselves should be considered as variables of the system, with the commutator relations viewed as their governing equations.

7 For readability, multiplets of equations, which can be obtained from each other by cyclic permutation of the spatial indices, will henceforth be represented by a single equation.
} 
that generically the full set of field equations can be interpreted as integrability conditions for the system governed by (5), (16) and (26). This system (wherein the commutator relations (8) will always be implicitly assumed to be valid) will be referred to as the JRB system. The emphasis is on 'generically', as the result is clearly false in certain overly simplified situations. Assuming for example a perfect fluid model with $\sigma_{a b}=\omega_{a}=\dot{u}_{a}=E_{a b}=H_{a b}=0$ and taking $\boldsymbol{u}$ to be the fluid's time-like eigenvector ${ }^{8}$, the only surviving JRB equations are (1) a single Bianchi equation (the conservation law for the matter density), (2) a single Ricci equation (the Raychaudhuri equation) and the 12 Jacobi equations for $n_{\alpha \beta}$ and $a_{\alpha}$, from which it is clearly impossible to derive the field equations.

In the case of a null-tetrad formalism (Newman-Penrose), the situation is less complicated, as the equations are usually already presented in their 'extended' form, as sets of 'NP equations' and 'Bianchi equations', where the former is just the set of second Cartan equations (13). In section 4, I will show how also the 'NP set' can be split into Jacobi equations and Ricci equations for one of the null congruences $(\boldsymbol{k})$, with the remaining field equations being the integrability conditions of the JRB set.

\section{The JRB system and the Einstein field equations in a $1+3$ setting}

In order to have no misunderstanding about the meaning of the 'JRB system', I first write out the equations explicitly. Where it is convenient, the expansion tensor $\Theta_{a b}=\sigma_{a b}+\frac{1}{3} \theta h_{a b}$ is used instead of the shear tensor and $\theta_{\alpha}=\Theta_{\alpha \alpha}$. A hybrid notation will be used, where boldface symbols refer to objects with greek indices, while the $3 \mathrm{D}$ operators $\cdot$ and $\times$ have their usual meaning, also when dealing with non-tensorial objects: for example $\boldsymbol{a} \cdot \boldsymbol{\omega}=a_{\alpha} \omega^{\alpha}$, $(\boldsymbol{\partial} \cdot \boldsymbol{n})_{\alpha}=\partial_{\beta} n_{\alpha}^{\beta}, \ldots$ (see also [35]). Note that no evolution equations are obtained for the variables $\dot{\boldsymbol{u}}$ and $\boldsymbol{\Omega}$ : this is not surprising as these are precisely the frame gauge source functions [14] reflecting the freedom of choice of the time-like congruence $\mathbf{u}$ and of the rotation rate of the spatial triad $\partial_{\alpha}$. In special circumstances [33], for example for a perfect fluid satisfying a barotropic equation of state, one may choose $\mathbf{u}$ to be the unique time-like eigenvector of the energy-momentum tensor and an evolution equation for $\dot{\mathbf{u}}$ is obtained from the $\left[\partial_{0}, \partial_{\alpha}\right] \rho$ commutator relations. A Fermi-propagated triad may be chosen to put $\boldsymbol{\Omega}=0$ or a co-rotating triad to put $\boldsymbol{\Omega}+\omega=0$. However, none of these gauge choices will be imposed in what follows.

\subsection{Jacobi equations}

Writing out the 16 Jacobi equations (16), one obtains 12 evolution equations,

$$
\begin{aligned}
& \partial_{0} \boldsymbol{\omega}=-\frac{1}{2}(\partial-\boldsymbol{a}) \times \dot{\boldsymbol{u}}+\frac{1}{2} \boldsymbol{n} \cdot \dot{\boldsymbol{u}}+\boldsymbol{\sigma} \cdot \boldsymbol{\omega}+\boldsymbol{\omega} \times \boldsymbol{\Omega}-\frac{2}{3} \theta \boldsymbol{\omega}, \\
& \partial_{0} \boldsymbol{a}=\frac{1}{2} \boldsymbol{\partial} \cdot \boldsymbol{\sigma}-\frac{1}{2}(\boldsymbol{\partial}-2 \boldsymbol{a}+\dot{\boldsymbol{u}}) \times(\boldsymbol{\omega}+\boldsymbol{\Omega})-\frac{1}{3} \partial \theta-\frac{1}{3} \theta(\boldsymbol{a}+\dot{\boldsymbol{u}})-\boldsymbol{\sigma} \cdot\left(\boldsymbol{a}-\frac{1}{2} \dot{\boldsymbol{u}}\right), \\
& \partial_{0} n_{12}=\frac{1}{2} \partial_{1}\left(\sigma_{31}-\omega_{2}-\Omega_{2}\right)-\frac{1}{2} \partial_{2}\left(\sigma_{23}+\omega_{1}+\Omega_{1}\right)+\frac{1}{2} \partial_{3}\left(\theta_{2}-\theta_{1}\right) \\
& +\left(n_{11}+n_{22}\right) \sigma_{12}+\left(n_{22}-n_{11}\right)\left(\omega_{3}+\Omega_{3}\right)-n_{12} \theta_{3} \\
& +\frac{1}{2} \dot{u}_{1}\left(\sigma_{31}-\omega_{2}-\Omega_{2}\right)-\frac{1}{2} \dot{u}_{2}\left(\sigma_{23}+\omega_{1}+\Omega_{1}\right)-\frac{1}{2} \dot{u}_{3}\left(\theta_{1}-\theta_{2}\right) \\
& +n_{31}\left(\sigma_{23}+\omega_{1}+\Omega_{1}\right)+n_{23}\left(\sigma_{31}-\omega_{2}-\Omega_{2}\right) \text {, } \\
& \partial_{0} n_{11}=-\left(\partial_{1}+\dot{u}_{1}\right)\left(\omega_{1}+\Omega_{1}\right)-\left(\partial_{2}+\dot{u}_{2}\right) \sigma_{31}+\left(\partial_{3}+\dot{u}_{3}\right) \sigma_{12} \\
& +\boldsymbol{\partial} \cdot \boldsymbol{\Omega}+2(\boldsymbol{a}+\dot{\boldsymbol{u}}) \cdot \boldsymbol{\omega}+\boldsymbol{\Omega} \cdot \dot{\boldsymbol{u}} \\
& +n_{11}\left(\theta_{1}-\theta_{2}-\theta_{3}\right)+2 n_{12}\left(\sigma_{12}+\omega_{3}+\Omega_{3}\right)+2 n_{31}\left(\sigma_{31}-\omega_{2}-\Omega_{2}\right)
\end{aligned}
$$

8 Using the Einstein field equations, this would then necessarily be an FLRW model. 
and 4 spatial 'divergence' equations,

$$
\begin{aligned}
& \partial \cdot \omega=(2 a+\dot{u}) \cdot \omega, \\
& \partial \cdot n=-\partial \times a+2 \Theta \cdot \omega+2 n \cdot a+2 \omega \times \Omega .
\end{aligned}
$$

\subsection{Ricci equations applied to $\boldsymbol{u}$}

Writing out (26) and replacing, as explained above, the Riemann tensor by its decomposition in terms of $T_{a b}, E_{a b}$ and $H_{a b}$, one obtains 18 independent equations, 3 of which are just the evolution equations (33) for the vorticity. The remaining set splits into six evolution equations for the expansion tensor, the trace being the Raychaudhuri equation,

$$
\partial_{0} \theta=-\frac{1}{3} \theta^{2}-\frac{1}{2}(\rho+3 p)-\sigma_{\alpha \beta} \sigma^{\alpha \beta}+2 \omega_{\alpha} \omega^{\alpha}+\boldsymbol{\partial} \cdot \dot{\boldsymbol{u}}+\dot{\boldsymbol{u}} \cdot(\dot{\boldsymbol{u}}-2 \boldsymbol{a})
$$

and

$$
\begin{aligned}
\partial_{0} \theta_{1}=\partial_{1} \dot{u}_{1}- & \frac{1}{9} \theta^{2}-\frac{1}{6}(\rho+3 p)-\sigma_{11}\left(\sigma_{11}+\frac{2}{3} \theta\right)-\sigma_{12}^{2}-\sigma_{31}^{2}+\omega_{2}^{2}+\omega_{3}^{2} \\
& +\dot{u}_{1}^{2}+\dot{u}_{2}\left(n_{31}-a_{2}\right)-\dot{u}_{3}\left(n_{12}+a_{3}\right)+2\left(\sigma_{12} \Omega_{3}-\sigma_{31} \Omega_{2}\right)+\frac{1}{2} \pi_{11}-E_{11},
\end{aligned}
$$

$$
\begin{aligned}
\partial_{0} \sigma_{12}=\partial_{(1} \dot{u}_{2)} & -\sigma_{12}\left(\theta_{1}+\theta_{2}\right)-\sigma_{31} \sigma_{23}+\sigma_{31} \Omega_{1}-\sigma_{23} \Omega_{2}+\left(\theta_{2}-\theta_{1}\right) \Omega_{3}+\dot{u}_{1} \dot{u}_{2}-\omega_{1} \omega_{2} \\
& -\frac{1}{2}\left(n_{31} \dot{u}_{1}-n_{23} \dot{u}_{2}\right)+\frac{1}{2}\left(n_{11}-n_{22}\right) \dot{u}_{3}+a_{(1} \dot{u}_{2)}+\frac{1}{2} \pi_{12}-E_{12} .
\end{aligned}
$$

There remain nine equations for the spatial derivatives of the vorticity components, which are equivalent to the nine 'div $\boldsymbol{\sigma}$ ' and 'curl $\boldsymbol{\sigma}$ ' equations of the $1+3$ covariant formalism,

$$
\begin{aligned}
\partial_{1} \omega_{2}=\partial_{1} \sigma_{31}- & \partial_{3} \theta_{1}+\left(\sigma_{23}+\omega_{1}\right)\left(n_{31}-a_{2}\right)-2 \dot{u}_{1} \omega_{2}+\frac{1}{2} \omega_{3}\left(n_{22}+n_{33}-n_{11}\right)+\frac{1}{2} q_{3}+H_{12} \\
& +\frac{1}{2} \sigma_{12}\left(n_{11}+3 n_{22}-n_{33}\right)+\left(n_{12}+a_{3}\right)\left(\theta_{1}-\theta_{3}\right)+2 \sigma_{31}\left(n_{23}-a_{1}\right), \\
\partial_{1} \omega_{3}=\partial_{2} \theta_{1}- & \partial_{1} \sigma_{12}+\left(\sigma_{23}-\omega_{1}\right)\left(n_{12}+a_{3}\right)-2 \dot{u}_{1} \omega_{3}-\frac{1}{2} \omega_{2}\left(n_{33}+n_{22}-n_{11}\right)-\frac{1}{2} q_{2}+H_{31} \\
& +\frac{1}{2} \sigma_{31}\left(n_{11}+3 n_{33}-n_{22}\right)+\left(n_{31}-a_{2}\right)\left(\theta_{1}-\theta_{2}\right)+2 \sigma_{12}\left(n_{23}+a_{1}\right), \\
\partial_{1} \omega_{1}=\partial_{3} \sigma_{12} & -\partial_{2} \sigma_{31}-\omega_{1} \dot{u}_{1}+\omega_{2}\left(\dot{u}_{2}+a_{2}-n_{31}\right)+\omega_{3}\left(\dot{u}_{3}+a_{3}+n_{12}\right)+H_{11} \\
& +\sigma_{31}\left(n_{31}+a_{2}\right)+\sigma_{12}\left(n_{12}-a_{3}\right)-2 n_{23} \sigma_{23} \\
& +\frac{1}{2} n_{11}\left(3 \theta_{1}-\theta\right)-\frac{1}{2}\left(n_{22}-n_{33}\right)\left(\theta_{2}-\theta_{3}\right) .
\end{aligned}
$$

Note that with (44), using $H_{\alpha}^{\alpha}=0$, the Jacobi equation (37) becomes an identity.

\subsection{Bianchi equations}

Writing out the Bianchi equations (14), one first obtains the four contracted equations, determining the evolution of $\rho$ and $q_{\alpha}$,

$$
\begin{aligned}
& \partial_{0} \rho=-(\rho+p) \theta-\boldsymbol{\partial} \cdot \boldsymbol{q}-2 \boldsymbol{q} \cdot(\dot{\boldsymbol{u}}-\boldsymbol{a})-\pi_{\alpha \beta} \sigma^{\alpha \beta}, \\
& \begin{aligned}
\partial_{0} q_{1}=(-\boldsymbol{\partial} p- & (\rho+p) \dot{\boldsymbol{u}}-\boldsymbol{\partial} \cdot \boldsymbol{\pi}+\boldsymbol{\pi} \cdot(3 \boldsymbol{a}-\dot{\boldsymbol{u}})-\boldsymbol{\Theta} \cdot \boldsymbol{q}-\theta \boldsymbol{q})_{1}-q_{2}\left(\omega_{3}-\Omega_{3}\right) \\
& \quad+q_{3}\left(\omega_{2}-\Omega_{2}\right)+\pi_{31} n_{12}-\pi_{12} n_{31}-\left(\pi_{22}-\pi_{33}\right) n_{23}+\left(n_{22}-n_{33}\right) \pi_{23} .
\end{aligned}
\end{aligned}
$$

Then follow the sets of ten 'dot E', 'dot H' and six 'div E', 'div H' equations, the appearance of which below is somewhat more complicated than the familiar (perfect fluid) one by the 
presence of the $q_{\alpha}$ and $\pi_{\alpha \beta}$ terms:

$$
\begin{aligned}
\partial_{0} E_{12}= & -\frac{1}{2} \partial_{2} q_{1}-\frac{1}{2} \partial_{0} \pi_{12}+\partial_{2} H_{23}-\partial_{3} H_{22}-\frac{1}{2}\left(\omega_{3}+\sigma_{12}\right)(\rho+p) \\
& +\frac{1}{4}\left(n_{22}-n_{33}-n_{11}\right) q_{3}+\frac{1}{2}\left(-n_{23}-a_{1}-\dot{u}_{1}\right) q_{2}-\frac{1}{2} \dot{u}_{2} q_{1} \\
& -\left(\Omega_{2}-2 \sigma_{31}+2 \omega_{2}\right) E_{23}+\left(\sigma_{23}+\Omega_{1}-\omega_{1}\right) E_{31}-\left(\theta_{2}+2 \theta_{3}\right) E_{12} \\
& +\left(2 \Omega_{3}+\sigma_{12}+\omega_{3}\right) E_{22}+\left(\Omega_{3}-\sigma_{12}-\omega_{3}\right) E_{33} \\
& +\left(\dot{u}_{2}-2 a_{2}-2 n_{31}\right) H_{23}-\frac{1}{2}\left(3 n_{11}+n_{22}-n_{33}\right) H_{12}-\left(a_{1}+\dot{u}_{1}+n_{23}\right) H_{31} \\
& +\left(a_{3}-n_{12}-2 \dot{u}_{3}\right) H_{22}+\left(n_{12}-a_{3}-\dot{u}_{3}\right) H_{33} \\
& -\frac{1}{2}\left(\left(\sigma_{12}+\omega_{3}+\Omega_{3}\right) \pi_{11}+\Omega_{2} \pi_{23}+\theta_{2} \pi_{12}-\Omega_{3} \pi_{22}-\left(\Omega_{1}-\sigma_{23}+\omega_{1}\right) \pi_{31}\right),
\end{aligned}
$$

$$
\begin{aligned}
\partial_{0} E_{11}= & -\frac{1}{2} \partial_{0} \pi_{11}-\frac{1}{2} \partial_{1} q_{1}+\frac{1}{6} \boldsymbol{\partial} \cdot \boldsymbol{q}+\partial_{2} H_{31}-\partial_{3} H_{12}+\frac{1}{6}\left(\theta-3 \theta_{1}\right)(\rho+p) \\
& -\frac{1}{3}\left(2 \dot{u}_{1}+a_{1}\right) q_{1}+\frac{1}{6}\left(a_{2}+2 \dot{u}_{2}-3 n_{31}\right) q_{2}+\frac{1}{6}\left(a_{3}+3 n_{12}+2 \dot{u}_{3}\right) q_{3} \\
& +\left(\sigma_{12}+\omega_{3}+2 \Omega_{3}\right) E_{12}-2 E_{23} \sigma_{23}+\left(\sigma_{31}-\omega_{2}-2 \Omega_{2}\right) E_{31} \\
& -\left(\theta_{2}+2 \theta_{3}\right) E_{11}+\left(\theta_{2}-\theta_{3}\right) E_{33}-\frac{3}{2} H_{11} n_{11}+\frac{1}{2}\left(n_{22}-n_{33}\right)\left(H_{22}-H_{33}\right) \\
& +2 H_{23} n_{23}-\left(n_{31}-2 \dot{u}_{2}+a_{2}\right) H_{31}-\left(n_{12}+2 \dot{u}_{3}-a_{3}\right) H_{12} \\
& -\frac{1}{6}\left(\sigma_{31}+3 \omega_{2}+6 \Omega_{2}\right) \pi_{31}-\frac{1}{6}\left(\sigma_{12}-3 \omega_{3}-6 \Omega_{3}\right) \pi_{12}+\frac{1}{3} \pi_{23} \sigma_{23} \\
& -\frac{1}{6}\left(2 \theta_{1}+\theta_{3}\right) \pi_{11}+\frac{1}{6}\left(\theta_{2}-\theta_{3}\right) \pi_{22},
\end{aligned}
$$

$$
\begin{aligned}
\partial_{0} H_{12}= & \partial_{3}\left(E_{22}+\frac{1}{6} \rho+\frac{1}{2} \pi_{11}\right)-\frac{1}{2} \partial_{1} \pi_{31}-\partial_{2} E_{23}+\frac{1}{2}\left(\sigma_{31}-3 \omega_{2}\right) q_{1}-\frac{1}{2} q_{3} \theta_{1} \\
& +\frac{1}{2}\left(3 n_{11}+n_{22}-n_{33}\right) E_{12}+\left(n_{23}+a_{1}+\dot{u}_{1}\right) E_{31}+\left(2 n_{31}-\dot{u}_{2}+2 a_{2}\right) E_{23} \\
& +\left(2 a_{3}-2 n_{12}-\dot{u}_{3}\right) E_{33}-\left(n_{12}+2 \dot{u}_{3}-a_{3}\right) E_{11} \\
& +\left(2 \sigma_{31}-2 \omega_{2}-\Omega_{2}\right) H_{23}+\left(\sigma_{23}-\omega_{1}+\Omega_{1}\right) H_{31}-\left(\theta_{2}+2 \theta_{3}\right) H_{12} \\
& +\left(H_{22}-H_{11}\right) \Omega_{3}+\left(\sigma_{12}+\omega_{3}\right)\left(H_{22}-H_{33}\right)-\frac{1}{2}\left(a_{3}+n_{12}\right)\left(\pi_{11}-\pi_{33}\right) \\
& +\frac{1}{4}\left(n_{33}-n_{11}-3 n_{22}\right) \pi_{12}+\left(a_{1}-n_{23}\right) \pi_{31}+\frac{1}{2}\left(a_{2}-n_{31}\right) \pi_{23},
\end{aligned}
$$

$$
\begin{aligned}
\partial_{0} H_{11}= & \partial_{3}\left(E_{12}-\frac{1}{2} \pi_{12}\right)-\partial_{2}\left(E_{31}-\frac{1}{2} \pi_{31}\right)-q_{1} \omega_{1}+\frac{1}{2}\left(\omega_{2}-\sigma_{31}\right) q_{2}+\frac{1}{2}\left(\sigma_{12}+\omega_{3}\right) q_{3} \\
& +\left(n_{12}-a_{3}+2 \dot{u}_{3}\right) E_{12}-2 E_{23} n_{23}+\left(n_{31}+a_{2}-2 \dot{u}_{2}\right) E_{31} \\
& +\frac{1}{2}\left(n_{33}-3 n_{11}-n_{22}\right)\left(E_{22}-\frac{1}{2} \pi_{22}\right)+\frac{1}{2}\left(n_{22}-3 n_{11}-n_{33}\right)\left(E_{33}-\frac{1}{2} \pi_{33}\right) \\
& +H_{22}\left(\theta_{2}+2 \theta_{3}\right)+H_{33}\left(\theta_{3}+2 \theta_{2}\right)+\left(\sigma_{12}+\omega_{3}+2 \Omega_{3}\right) H_{12} \\
& +\left(\sigma_{31}-\omega_{2}-2 \Omega_{2}\right) H_{31}-2 \sigma_{23} H_{23}+\frac{1}{2}\left(n_{22}-n_{33}\right) \pi_{22}+n_{23} \pi_{23} \\
& -\frac{1}{2}\left(n_{12}-a_{3}\right) \pi_{12}-\frac{1}{2}\left(n_{31}+a_{2}\right) \pi_{31}
\end{aligned}
$$

and

$$
\begin{aligned}
(\boldsymbol{\partial} \cdot \boldsymbol{E})_{3}= & \left(\frac{1}{3} \boldsymbol{\partial} \rho-\frac{1}{2} \boldsymbol{\partial} \cdot \boldsymbol{\pi}+\frac{3}{2} \boldsymbol{\omega} \times \boldsymbol{q}+\frac{1}{2} \boldsymbol{\Theta} \cdot \boldsymbol{q}+3 \boldsymbol{E} \cdot \boldsymbol{a}-3 \boldsymbol{H} \cdot \boldsymbol{\omega}+\frac{3}{2} \boldsymbol{\pi} \cdot \boldsymbol{a}\right)_{3} \\
& +n_{31} E_{23}-n_{23} E_{31}+\left(n_{11}-n_{22}\right) E_{12}+\left(E_{22}-E_{11}\right) n_{12} \\
& +\sigma_{12}\left(H_{22}-H_{11}\right)+\left(\theta_{1}-\theta_{2}\right) H_{12}+\sigma_{31} H_{23}-\sigma_{23} H_{31}-\frac{1}{2} n_{23} \pi_{31}+\frac{1}{2} n_{31} \pi_{23} \\
& +\frac{1}{2}\left(n_{12}-3 a_{3}\right) \pi_{22}-\frac{1}{2}\left(n_{12}+3 a_{3}\right) \pi_{11}+\frac{1}{2}\left(n_{11}-n_{22}\right) \pi_{12}, \\
(\boldsymbol{\partial} \cdot \boldsymbol{H})_{3}= & \left(-\frac{1}{2} \boldsymbol{\partial} \times \boldsymbol{q}+(\rho+p) \boldsymbol{\omega}+\frac{1}{2} \boldsymbol{a} \times \boldsymbol{q}+\frac{1}{2} \boldsymbol{n} \cdot \boldsymbol{q}+3 \boldsymbol{E} \cdot \boldsymbol{\omega}+3 \boldsymbol{H} \cdot \boldsymbol{a}-\frac{1}{2} \boldsymbol{\pi} \cdot \boldsymbol{\omega}\right)_{3} \\
& +\left(\theta_{2}-\theta_{1}\right) E_{12}-\sigma_{31} E_{23}+\sigma_{23} E_{31}+\sigma_{12}\left(E_{11}-E_{22}\right) \\
& +n_{31} H_{23}-n_{23} H_{31}+\left(n_{11}-n_{22}\right) H_{12}+n_{12}\left(H_{22}-H_{11}\right) \\
& -\frac{1}{2}\left(\theta_{1}-\theta_{2}\right) \pi_{12}+\frac{1}{2} \sigma_{23} \pi_{31}-\frac{1}{2} \sigma_{31} \pi_{23}+\frac{1}{2} \sigma_{12}\left(\pi_{11}-\pi_{22}\right) .
\end{aligned}
$$


To sum up, the JRB system consists of the following independent sets: 15 Jacobi equations (12 evolution equations (33)-(36) and 3 'div $\boldsymbol{n}$ ' equations (38)), 15 Ricci equations (6 evolution equations (39)-(41) and 9 equations (42)-(44) for the spatial derivatives of the vorticity) and 20 Bianchi equations (14 evolution equations (45), (46), (47)-(50) and 6 'div $\boldsymbol{E}$ ', 'div $\boldsymbol{H}$ ' equations (51), (52)).

\subsection{Einstein field equations}

We now write out the Einstein field equations, simplifying the left-hand side of (1) by using all the algebraic information in the Jacobi and Ricci equations. The $(0 \alpha)$ components become then identically satisfied, while the (00) equation reduces to the trace of the $(\alpha \alpha)$ field equations. We write the remaining six equations as $\mathcal{Y}_{\alpha \beta}=0$, with

$$
\begin{aligned}
\mathcal{Y}_{11} \equiv \partial_{2}\left(n_{31}\right. & \left.+a_{2}\right)-\partial_{3}\left(n_{12}-a_{3}\right)+\theta_{2} \theta_{3}-\sigma_{23}{ }^{2}+\omega_{1}\left(\omega_{1}-2 \Omega_{1}\right)-a_{\alpha} a^{\alpha} \\
& +\frac{1}{4} n_{11}\left(2 n_{22}+2 n_{33}-3 n_{11}\right)+\frac{1}{4}\left(n_{22}-n_{33}\right)^{2}+n_{23}{ }^{2} \\
& -n_{12}\left(n_{12}-2 a_{3}\right)-n_{31}\left(n_{31}+2 a_{2}\right)+E_{11}-\frac{1}{3} \rho+\frac{1}{2} \pi_{11}
\end{aligned}
$$

and

$$
\begin{aligned}
\mathcal{Y}_{12} \equiv \frac{1}{2} \partial_{1}\left(n_{31}\right. & \left.+a_{2}\right)-\frac{1}{2} \partial_{2}\left(n_{23}-a_{1}\right)-\frac{1}{2} \partial_{3}\left(n_{11}-n_{22}\right)+\omega_{1} \Omega_{2}+\omega_{2} \Omega_{1}-\omega_{2} \omega_{1} \\
& -\sigma_{31} \sigma_{23}+\theta_{3} \sigma_{12}+n_{12}\left(n_{11}+n_{22}-n_{33}\right)+2 n_{31} n_{23} \\
& -n_{31} a_{1}+n_{23} a_{2}+a_{3}\left(n_{11}-n_{22}\right)-E_{12}-\frac{1}{2} \pi_{12} .
\end{aligned}
$$

It might appear odd that the electric part $E_{a b}$ of the Weyl tensor shows up on the left-hand side of (1), but this is of course a consequence of the fact that we have eliminated the evolution of the shear in favour of $E_{a b}$ by means of the Ricci equations (40) and (41).

\subsection{Integrability conditions for the JRB system}

The JRB system provides us with expressions for all derivatives of the vorticity, in terms of spatial derivatives of the expansion tensor and the acceleration. It is relatively easy to verify that the integrability conditions for these equations are identically satisfied under the Einstein field equations (see also [34], where the propagation of the constraints under the evolution equations was demonstrated for a barotropic perfect fluid, or [32] for a general energy-momentum tensor and a non-rotating congruence). That the field equations can also be viewed as integrability conditions for the JRB system (provided the $\boldsymbol{u}$ congruence is generic) is not evident at all, but can be seen by evaluating the following set of commutators:

$$
\begin{aligned}
& {\left[\partial_{0}, \partial_{1}\right]\left(\sigma_{31}+\omega_{2}\right)+\left[\partial_{0}, \partial_{3}\right] \theta_{1}+\left[\partial_{3}, \partial_{1}\right] \dot{u}_{1},} \\
& {\left[\partial_{0}, \partial_{1}\right]\left(\sigma_{12}+\omega_{3}\right)-\left[\partial_{0}, \partial_{2}\right] \theta_{1}+\left[\partial_{1}, \partial_{2}\right] \dot{u}_{1},} \\
& {\left[\partial_{1}, \partial_{2}\right]\left(\sigma_{23}+\omega_{1}\right)+\left[\partial_{2}, \partial_{3}\right]\left(\sigma_{12}-\omega_{3}\right)+\left[\partial_{3}, \partial_{1}\right] \theta_{2} .}
\end{aligned}
$$

From the resulting expressions (and from similar ones obtained by cyclic permutation of the indices), all second-order derivatives can be eliminated, leading to a homogeneous system of first-order equations with the following simple structure:

$$
\begin{aligned}
& {\left[\begin{array}{lll}
\dot{u}_{3} & 0 & -\dot{u}_{2} \\
-\dot{u}_{3} & \dot{u}_{1} & 0 \\
0 & -\dot{u}_{1} & \dot{u}_{2}
\end{array}\right]\left[\begin{array}{l}
\mathcal{Y}_{12} \\
\mathcal{Y}_{23} \\
\mathcal{Y}_{31}
\end{array}\right]=0,} \\
& \operatorname{diag}\left(\dot{u}_{1}, \dot{u}_{2}, \dot{u}_{3}\right)\left[\begin{array}{l}
\mathcal{Y}_{33} \\
\mathcal{Y}_{11} \\
\mathcal{Y}_{22}
\end{array}\right]-\left[\begin{array}{lll}
0 & 0 & \dot{u}_{3} \\
\dot{u}_{1} & 0 & 0 \\
0 & \dot{u}_{2} & 0
\end{array}\right]\left[\begin{array}{l}
\mathcal{Y}_{12} \\
\mathcal{Y}_{23} \\
\mathcal{Y}_{31}
\end{array}\right]=0,
\end{aligned}
$$




$$
\begin{aligned}
& \operatorname{diag}\left(\dot{u}_{2}, \dot{u}_{3}, \dot{u}_{1}\right)\left[\begin{array}{l}
\mathcal{Y}_{33} \\
\mathcal{Y}_{11} \\
\mathcal{Y}_{22}
\end{array}\right]-\left[\begin{array}{lll}
0 & -\dot{u}_{3} & 0 \\
0 & 0 & \dot{u}_{1} \\
-\dot{u}_{2} & 0 & 0
\end{array}\right]\left[\begin{array}{l}
\mathcal{Y}_{12} \\
\mathcal{Y}_{23} \\
\mathcal{Y}_{31}
\end{array}\right]=0, \\
& {\left[\begin{array}{lll}
\sigma_{23}+\omega_{1} & 0 & -\sigma_{23}+\omega_{1} \\
0 & -\sigma_{12}+\omega_{3} & \sigma_{12}+\omega_{3} \\
-\sigma_{31}+\omega_{2} & \sigma_{31}+\omega_{2} & 0
\end{array}\right]\left[\begin{array}{l}
\mathcal{Y}_{33} \\
\mathcal{Y}_{11} \\
\mathcal{Y}_{22}
\end{array}\right]} \\
& +\left[\begin{array}{lll}
-\sigma_{31}-\omega_{2} & \theta_{2}-\theta_{3} & \sigma_{12}-\omega_{3} \\
\theta_{1}-\theta_{2} & \sigma_{31}-\omega_{2} & -\sigma_{23}-\omega_{1} \\
\sigma_{23}-\omega_{1} & -\sigma_{12}-\omega_{3} & \theta_{3}-\theta_{1}
\end{array}\right]\left[\begin{array}{l}
\mathcal{Y}_{12} \\
\mathcal{Y}_{23} \\
\mathcal{Y}_{31}
\end{array}\right]=0 .
\end{aligned}
$$

Aligning the $\partial_{3}$ axis with the acceleration $\left(\dot{u}_{1}=\dot{u}_{2}=0\right)$, it is clear that, for non-vanishing acceleration, $\mathcal{Y}_{12}=\mathcal{Y}_{23}=\mathcal{Y}_{31}=\mathcal{Y}_{11}=\mathcal{Y}_{22}=0$, which when substituted in (61) implies $\left(\sigma_{31}-\omega_{2}\right) \mathcal{Y}_{33}=\left(\sigma_{23}+\omega_{1}\right) \mathcal{Y}_{33}=0$. It follows that $\mathcal{Y}_{33}=0$ unless $\sigma_{\alpha 3}+\omega_{\alpha 3}=0$, meaning that $\dot{\boldsymbol{u}}$ is an eigenvector of $\sigma_{a b}+\omega_{a b}$ :

Theorem 1. If $\dot{\boldsymbol{u}} \neq 0$ is not an eigenvector of $\sigma_{a b}+\omega_{a b}$, then the Einstein field equations are the integrability conditions of the JRB system.

Note that this theorem is also an immediate consequence of the property that the curvature tensor for a given metric connection $\nabla$ is the unique tensor obeying

- the symmetries (18)

- the first Bianchi equations (14)

- the second Bianchi equations (17)

- the Ricci equations (26) for a generic $\boldsymbol{u}$ congruence.

A simple covariant proof of this uniqueness property is obtained by introducing the difference $\boldsymbol{A}$ of two such tensors and contracting (17) with $u^{b}$, implying

$$
A_{a b[c d} u_{; e]}^{b}=0 .
$$

By (26) one has $A_{a b c d} u^{d}=0$ and hence a further contraction of (62) with $u^{e}$ implies $A_{a b c d} \dot{u}^{d}=0$. Defining a tetrad with $\partial_{0}=\boldsymbol{u}$ and $\partial_{3}=\dot{\boldsymbol{u}}$, it follows that the only possible non-zero component of $\boldsymbol{A}$ is given by $A_{1212}\left(=A_{2121}=-A_{1221}=-A_{2112}\right)$. Choosing in (62) $($ acde $)=(1123)$ or $(2213)$ gives then

$$
A_{2121} u_{; 3}^{1}=A_{1212} u_{; 3}^{2}=0,
$$

which indeed implies $\boldsymbol{A}=0$, unless $\dot{u}_{[c} u_{a] ; b} \dot{u}^{b}=0$, i.e. unless $\dot{\boldsymbol{u}}$ is an eigenvector of $\sigma_{a b}+\omega_{a b}$.

A similar property - not involving the acceleration of the time-like congruence-can be obtained by considering the integrability conditions of the second Bianchi equations: eliminating the second-order derivatives from the commutator relations $\left[\partial_{0}, \partial_{1}\right] H_{11}+$ $\left[\partial_{0}, \partial_{2}\right] H_{12}+\left[\partial_{0}, \partial_{3}\right] H_{13}-\frac{1}{6}\left[\partial_{2}, \partial_{3}\right] \rho-\left[\partial_{3}, \partial_{1}\right] E_{12}-\left[\partial_{1}, \partial_{2}\right] E_{31}-\left[\partial_{2}, \partial_{3}\right] E_{11}$ and $\left[\partial_{0}, \partial_{1}\right] E_{11}+\left[\partial_{0}, \partial_{2}\right] E_{12}+\left[\partial_{0}, \partial_{3}\right] E_{13}-\frac{1}{3}\left[\partial_{0}, \partial_{3}\right] \rho+\left[\partial_{3}, \partial_{1}\right] H_{23}+\left[\partial_{1}, \partial_{2}\right] H_{33}+\left[\partial_{2}, \partial_{3}\right] H_{31}$ again results in a homogeneous system for the $\mathcal{Y}_{\alpha \beta}$ variables:

$$
\begin{aligned}
& {\left[\begin{array}{lll}
-\mathcal{I}_{23} & 0 & \mathcal{I}_{23} \\
\mathcal{I}_{31} & -\mathcal{I}_{31} & 0 \\
0 & \mathcal{I}_{12} & -\mathcal{I}_{12}
\end{array}\right]\left[\begin{array}{l}
\mathcal{Y}_{33} \\
\mathcal{Y}_{11} \\
\mathcal{Y}_{22}
\end{array}\right]=\left[\begin{array}{lll}
-\mathcal{I}_{31} & \mathcal{I}_{22}-\mathcal{I}_{33} & \mathcal{I}_{12} \\
\mathcal{I}_{23} & -\mathcal{I}_{12} & \mathcal{I}_{33}-\mathcal{I}_{11} \\
\mathcal{I}_{11}-\mathcal{I}_{22} & \mathcal{I}_{31} & -\mathcal{I}_{23}
\end{array}\right]\left[\begin{array}{l}
\mathcal{Y}_{12} \\
\mathcal{Y}_{23} \\
\mathcal{Y}_{31}
\end{array}\right],} \\
& {\left[\begin{array}{lll}
0 & -\mathcal{J}_{21} & \mathcal{J}_{12} \\
-\mathcal{J}_{13} & \mathcal{J}_{31} & 0 \\
\mathcal{J}_{23} & 0 & -\mathcal{J}_{32}
\end{array}\right]\left[\begin{array}{l}
\mathcal{Y}_{33} \\
\mathcal{Y}_{11} \\
\mathcal{Y}_{22}
\end{array}\right]=\left[\begin{array}{lll}
\mathcal{J}_{22}-\mathcal{J}_{11} & -\mathcal{J}_{13} & \mathcal{J}_{23} \\
-\mathcal{J}_{32} & \mathcal{J}_{12} & \mathcal{J}_{11}-\mathcal{J}_{33} \\
\mathcal{J}_{31} & \mathcal{J}_{33}-\mathcal{J}_{22} & -\mathcal{J}_{21}
\end{array}\right]\left[\begin{array}{l}
\mathcal{Y}_{12} \\
\mathcal{Y}_{23} \\
\mathcal{Y}_{31}
\end{array}\right]} \\
& \text { with } \mathcal{I}_{a b}=E_{a b}-\frac{1}{2} \pi_{a b} \text { and } \mathcal{J}_{a b}=H_{a b}-\frac{1}{2} \eta_{a b c d} q^{c} u^{d} .
\end{aligned}
$$


Generically, the corresponding $6 \times 6$ determinant is different from zero and the system will again have only the zero-solution. Except for a few particular cases it is not easy however to provide a simple geometric characterization of the non-vanishing of this determinant. It is clear that the left-hand side of (64) is zero in an $\mathcal{I}$ eigenframe, such that $\mathcal{Y}_{12}=\mathcal{Y}_{23}=\mathcal{Y}_{31}=0$ when $\mathcal{I}$ is non-degenerate. If $\mathcal{I}$ and $\boldsymbol{H}$ commute, a common eigenframe can therefore be constructed in which clearly also $\mathcal{Y}_{11}=\mathcal{Y}_{22}=\mathcal{Y}_{33}=0$ provided $q_{1} q_{2} q_{3} \neq 0$ (this will happen for example when $\mathbf{u}$ is the time-like eigenvector of a perfect fluid energy-momentum tensor for which $\mathbf{E}$ and $\mathbf{H}$ commute and hence a fortiori occurs for all purely electric or purely magnetic perfect fluids). Hence, we obtain

Theorem 2. When $[\mathcal{I}, \boldsymbol{H}]=0, \mathcal{I}_{\alpha \beta}$ is not degenerate and $\boldsymbol{q}$ is not parallel to one of the eigenblades of $\boldsymbol{H}$, then the Einstein field equations are the integrability conditions of the JRB system.

On the other hand, it is easy to verify that the Einstein field equations cannot be obtained from (64) and (65) when for example $\boldsymbol{q}=0$, as then the matrix on the left-hand side of (65) is singular.

\section{The case of a null congruence}

When the congruence, with respect to which the Ricci equations are constructed, is null, one can construct a Newman-Penrose tetrad $(\boldsymbol{m}, \overline{\boldsymbol{m}}, \boldsymbol{\ell}, \boldsymbol{k})$ with $\boldsymbol{k}=\boldsymbol{u}$ : the equations are then usually presented [28, 19] in their 'already extended' form, as the set of 'NP equations'these being just the second Cartan equations (13) — and the second Bianchi equations. We will refer to the two sets by their numbering in [19] as $\mathrm{NP}_{1}-\mathrm{NP}_{18}$ and $B_{1}-B_{11}$, with the latter being just the set of integrability conditions of the former. One may again isolate from the NP equations the subsets of Jacobi and Ricci equations and ask whether the remaining part of the NP equations (the 'remaining' field equations) is obtainable as the integrability conditions of the JRB set. An explicit evaluation of (15) shows that the Jacobi-part of the NP equations is expressed by the 16 independent equations (4 imaginary and 6 complex)

$$
\begin{aligned}
& \overline{\mathrm{NP}_{1}}-\mathrm{NP}_{1}, \overline{\mathrm{NP}_{14}}-\mathrm{NP}_{14}, \overline{\mathrm{NP}_{12}}+\overline{\mathrm{NP}_{8}}-\mathrm{NP}_{12}-\mathrm{NP}_{8}, \\
& \overline{\mathrm{NP}_{6}}-\mathrm{NP}_{6}+\mathrm{NP}_{8}-\overline{\mathrm{NP}_{8}}, \mathrm{NP}_{16}-\overline{\mathrm{NP}_{7}}, \mathrm{NP}_{17}+\mathrm{NP}_{8}, \\
& 2 \mathrm{NP}_{4}-\overline{\mathrm{NP}_{3}}-\overline{\mathrm{NP}_{11}}, 2 \mathrm{NP}_{5}-\mathrm{NP}_{3}+\mathrm{NP}_{11}, \\
& 2 \mathrm{NP}_{15}-\overline{\mathrm{NP}_{9}}-\overline{\mathrm{NP}_{13}}, 2 \mathrm{NP}_{18}+\mathrm{NP}_{9}-\mathrm{NP}_{13} .
\end{aligned}
$$

The Ricci equations (25) for $\boldsymbol{k}$ on the other hand give rise to 18 independent equations ( 1 real, 1 imaginary and 8 complex)

$$
\begin{aligned}
& \mathrm{NP}_{1}, \mathrm{NP}_{2}, \mathrm{NP}_{3}, \mathrm{NP}_{11}, \mathrm{NP}_{16}, \mathrm{NP}_{17}, \overline{\mathrm{NP}_{4}}+\mathrm{NP}_{5}, \\
& \mathrm{NP}_{15}-\overline{\mathrm{NP}_{18}}, \overline{\mathrm{NP}_{6}}+\mathrm{NP}_{6}, \overline{\mathrm{NP}_{12}}-\mathrm{NP}_{12},
\end{aligned}
$$

which together with (66) reduce the remaining field equations to a set of 6 equations ( 2 real and 2 complex),

$$
\mathrm{NP}_{10}, \mathrm{NP}_{13}, \mathrm{NP}_{12}+\overline{\mathrm{NP}_{12}}, \mathrm{NP}_{14}+\overline{\mathrm{NP}_{14}}
$$

(or any set equivalent to it under (66) and (67)). Considering the linear combinations of commutators $[\bar{\delta}, \delta] \kappa+[\delta, D] \rho-[\bar{\delta}, D] \sigma,[\Delta, D] \rho+[\bar{\delta}, \Delta] \kappa-[\bar{\delta}, D] \tau,[\Delta, D] \alpha-[\bar{\delta}, D] \gamma+$ $[\bar{\delta}, \Delta] \epsilon$ and $[\Delta, D] \beta-[\delta, D] \gamma+[\delta, \Delta] \epsilon$, one obtains then, using (66) and (67), the equations $\kappa \mathrm{NP}_{13}, \kappa \mathrm{NP}_{12}, \bar{\kappa} \mathrm{NP}_{14}-\kappa \mathrm{NP}_{10}$ and

$$
D\left(\mathrm{NP}_{13}\right)+\bar{\sigma} \overline{\mathrm{NP}_{13}}-(\rho-2 \epsilon) \mathrm{NP}_{13}-2(\pi+\tau) \mathrm{NP}_{12}+2 \kappa \mathrm{NP}_{10},
$$

from which, provided $\kappa \neq 0,(68)$ readily follows. 
Theorem 3. If $\kappa=-k_{a ; b} m^{a} k^{b} \neq 0$, then the Newman-Penrose equations $\mathrm{NP}_{10}, \mathrm{NP}_{13}$, $\mathrm{NP}_{12}+\overline{\mathrm{NP}_{12}}$ and $\mathrm{NP}_{14}+\overline{\mathrm{NP}_{14}}$ are the integrability conditions of the remaining NewmanPenrose equations and Bianchi equations.

\section{Conclusion}

Embedding the $1+3$ covariant equations in an extended tetrad formalism [13] leads to redundancies, forcing one to make a choice among the sets of Jacobi, Ricci, Bianchi equations and Einstein field equations (whereby with 'Ricci equations' we mean the full set of Ricci equations applied to the tangent vectorfield of a single time-like congruence). It is shown that a minimal set of equations can consist of 15 Jacobi and 15 Ricci equations, together with the 20 Bianchi equations, in which the Riemann tensor is defined in terms of trace-free and symmetric tensors $E_{a b}, H_{a b}$ and a symmetric tensor $T_{a b}$. The Einstein field equations arise then as the integrability conditions for this set, if one chooses the time-like congruence such that the acceleration is not zero and is not an eigenvector of $\sigma_{a b}+\omega_{a b}$, a condition which is obviously violated when $\boldsymbol{u}$ is the 4-velocity for pressure-free matter. It remains to be seen whether this alternative view of the Einstein equations, as integrability conditions of an extended system, has any useful applications at all, for example, in the area of numerical relativity. In the case of a null congruence $\boldsymbol{u}=\boldsymbol{k}$, an analogous result is obtained provided that the associated Newman-Penrose coefficient $\kappa=-k_{a ; b} m^{a} k^{b}$ is non-vanishing.

\section{Acknowledgments}

I thank Stanley Deser, Con Lozanovski and Lode Wylleman for comments and suggestions for improvement. All calculations were performed with the aid of the Maple symbolic algebra package.

\section{References}

[1] Ashtekar A 1986 Phys. Rev. Lett. 572244

[2] Capovilla R, Dell J, Jacobson T and Mason L 1991 Class. Quantum Grav. 841

[3] Deser S and Isham C 1976 Phys. Rev. D 142505

[4] Dunsby P K S, Bassett B A and Ellis G F R 1997 Class. Quantum Grav. 141215

[5] Cahen M, Debever R and Defrise L 1967 J. Math. Mech. 16761

[6] Costa L F O and Herdeiro C A R 2008 Phys. Rev. D 78024021

[7] Edgar S B 1980 Gen. Rel. Grav. 12347

[8] Edgar S B 1992 Gen. Rel. Grav. 241267

[9] Ellis G F R 1967 J. Math. Phys. 81171

[10] Ellis G F R and MacCallum M A H 1969 Commun. Math. Phys. 12108

[11] Ellis G F R 1971 General Relativity and Cosmology: Proc. Int. School of Physics 'Enrico Fermi' vol 47 ed R K Sachs (New York: Academic)

[12] Ellis G F R and Bruni M 1989 Phys. Rev. D 401804

[13] Ellis GFR, Maartens R and MacCallum M A H 2012 Relativistic Cosmology (Cambridge: Cambridge University Press)

[14] Friedrich H 1986 Commun. Math. Phys. 107587

[15] Friedrich H 1996 Class. Quantum Grav. 131451

[16] Hawking S W 1966 Astrophys. J. 145544

[17] Jantzen R T, Carini P and Bini D 1992 Ann. Phys., NY 2151

[18] Karlhede A 1980 Gen. Rel. Grav. 12693

[19] Kramer D, Stephani H, MacCallum M A H, Hoenselaers C and Herlt E 2003 Exact Solutions of Einstein's Field Equations (Cambridge: Cambridge University Press)

[20] Maartens R 1997 Phys. Rev. D 55463 
[21] Maartens R, Lesame W M and Ellis G F R 1998 Class. Quantum Grav. 151005

[22] Maartens R and Bassett B A 1998 Class. Quantum Grav. 15705

[23] MacCallum M A H 1971 Cosmological Models from a Geometric Point of View (Cargese Lectures in Physics vol 6) ed E Schatzman (New York: Gordon and Breach) p 61

[24] MacCallum M A H 1998 Integrability in tetrad formalisms and conservation in cosmology arXiv:gr-qc/9806003

[25] Papapetrou A 1970 Ann. Inst. Henri Poincaré 13271

[26] Papapetrou A 1971 C.R. Acad. Sci., Paris A 2721537

[27] Papapetrou A 1971 C.R. Acad. Sci., Paris A 2721613

[28] Penrose R and Rindler W 1986 Spinors and Space-Time (Cambridge: Cambridge University Press)

[29] Plebanski J F 1977 J. Math. Phys. 182511

[30] Shinkai H and Yoneda G 2000 Class. Quantum Grav. 174799

[31] Tsagas C G, Challinor A and Maartens R 2008 Phys. Rep. 46561

[32] Uggla C, van Elst H, Wainwright J and Ellis G F R 2003 Phys. Rev. D 6810302

[33] van Elst H and Uggla C 1997 Class. Quantum Grav. 142673

[34] van Elst H 1996 PhD Thesis Queen Mary and Westfield College, London

[35] Van den Bergh N and Wylleman L 2004 Class. Quantum Grav. 212291

[36] van Putten M H D M and Eardley D M 1996 Phys. Rev. D 533056

[37] Velden T 1997 Diplomarbeit University of Bielefeld

[38] Wainwright J and Ellis G 1997 Dynamical Systems in Cosmology (Cambridge: Cambridge University Press) 